\title{
FACTEURS BIOLOGIQUES A PRENDRE EN COMPTE DANS LA CONCEPTION DES OUVRAGES DE FRANCHISSEMENT, NOTIONS D'OBSTACLES A LA MIGRATION
}

\author{
M. LARINIER
}

CSP-CEMAGREF, GHAAPPE - Institut de Mécanique des Fluides Avenue du Professeur Camille Soula - 31400 TOULOUSE

\section{PERFORMANCES DE NAGE DES POISSONS ET VITESSES DANS LES DISPOSITIFS DE FRANCHISSEMENT}

\subsection{Les différents niveaux d'activité de nage}

On distingue généralement chez le poisson (BLAXTER, 1969 ; BELL, 1986 ; WEBB, 1975) plusieurs niveaux d'activité de nage qui font appel à différents types de muscles:

- l'activité de croisière ("routine", "cruising" ou "sustained activity level" selon les auteurs), susceptible d'être maintenue pendant des heures sans engendrer de modifications physiologiques profondes de son organisme, faisant appel à des mécanismes d'activité musculaire aérobies (muscles "rouges").

- l'activité de sprint ou de pointe ("sprint" ou "burst activity level"), résultant d'un effort continu et intense ne pouvant être maintenue au-delà d'un certain temps (de quelques secondes à quelques dizaines de secondes suivant la taille de l'individu et la température de l'eau). On peut rattacher à ce niveau d'activité l'accélération brutale ("darting", "fast-start activity") et le saut, activités explosives de très courte durée. La puissance musculaire mise en oeuvre est pratiquement totalement assurée par des mécanismes anaérobies (au niveau des muscles "blancs") qui se traduisent par la décomposition du glycogène musculaire en acide lactique. Si les réactions anaérobies permettent de générer très rapidement une grande puissance musculaire, elles mettent néanmoins en jeu une énergie limitée dans la mesure où les réserves en glycogène des muscles sont limitées et où la concentration en acide lactique, à partir d'un certain seuil, a tendance à inhiber en retour la contraction musculaire.

- l'activité soutenue ("sustained", "prolonged activity level" selon les auteurs). C'est une activité de nage pouvant être maintenue pendant plusieurs minutes, mais qui à terme entraîne la fatigue du poisson. Cette activité de nage fait appel dans des proportions variables aux différents mécanismes aérobies et anaérobies, et d'autant plus aux mécanismes anaérobies que l'intensité de l'effort est plus grande.

\subsection{Vitesses de nage et endurance}

Un des principaux facteurs à prendre en compte dans la conception des dispositifs de franchissement est la capacité de nage des migrateurs concernés, qui s'exprime en termes de vitesse de nage et d'endurance, temps pendant lequel le poisson peut soutenir cette vitesse de nage.

La propulsion de la plupart des espèces dans leur activité de migration (en particulier le franchissement des obstacles) est assurée par l'ondulation du corps et de la nageoire caudale.

Des expérimentations (WARDLE, 1975) ont montré que la distance parcourue (A) par le poisson à chaque ondulation du corps peut varier entre 0.6 et 0.8 fois sa longueur $(L)$. La vitesse de nage peut donc s'exprimer sous la forme : 


$$
V=A f
$$

où $f$ est la fréquence de l'ondulation du corps et de la nageoire caudale (nombre d'ondulations par seconde).

La vitesse maximale de nage est donc fonction de la fréquence maximale du battement de la nageoire caudale. Cette fréquence maximale est limitée par le temps minimum (t) séparant deux contractions des muscles paravertébraux assurant la propulsion du poisson. D'où l'expression (WARDLE, 1975) en prenant pour A la valeur moyenne 0.7 :

$$
\mathrm{V}=0.7 \mathrm{~L} / 2 \mathrm{t}
$$

Des expérimentations ont montré que le temps séparant deux contractions musculaires successives était beaucoup plus faible pour les individus de petite taille. La contraction des muscles "blancs" anaérobies est d'autre part très sensible à la température, le temps minimum séparant deux contractions musculaires décroissant avec la température (WARDLE, 1975).

La vitesse maximale de nage dépend donc avant tout de la longueur du poisson et de la température.

L'endurance dépend de la réserve en glycogène stockée dans les muscles. Cette réserve est utilisée dès que le poisson dépasse sa vitesse de croisière et le rythme de déplétion est fonction de la vitesse de nage et de la température.

L'endurance dépend, elle aussi, de la longueur du poisson, de sa morphologie (relation poids-longueur, pourcentage de masse musculaire) et de la température.

Des études expérimentales effectuées en Grande-Bretagne (WARDLE, 1980 ; ZHOU, 1982 ; BEACH, 1984) ont permis d'obtenir un certain nombre d'expressions empiriques donnant les relations existant entre vitesses de nage, endurance, température, taille et morphologie du poisson.

On a porté à partir de ces expressions empiriques sur les figures 1 et 2 les vitesses maximales de nage ainsi que l'endurance afférentes à ces vitesses de nage pour différentes températures et différentes tailles (d'après BEACH, 1984).

On a porté sur la figure 3 la relation vitesse de nage-endurance pour différentes tailles de poissons et pour plusieurs températures.

Ces résultats semblent être en parfaite concordance avec les données sur les vitesses maximales de nage proposées par différents auteurs (BELL, 1986) : $6 \mathrm{~m} / \mathrm{s}$ à plus de $8 \mathrm{~m} / \mathrm{s}$ pour le saumon, $3 \mathrm{~m} / \mathrm{s}$ à $4 \mathrm{~m} / \mathrm{s}$ pour la truite, $4 \mathrm{~m} / \mathrm{s}$ à plus de $5 \mathrm{~m} / \mathrm{s}$ pour l'alose dans des conditions thermiques favorables.

Ils ont l'intérêt de mettre clairement en évidence l'importance fondamentale du facteur thermique et de la taille du poisson sur la vitesse maximale de nage et surtout le rôle primordial de la taille du poisson sur l'endurance.

Les vitesses maximales de nage, pour un même poisson, peuvent varier dans le rapport de 1 à 2 suivant la température.

On notera que pour un poisson de taille donnée, une augmentation de la température se traduit par une diminution importante de l'endurance. Cette réduction s'explique par le fait qu'à une température plus élevée correspond une vitesse maximale plus importante et partant une déplétion plus rapide des réserves en glycogène des muscles, d'où une endurance plus faible.

Les vitesses de croisière diminuent rapidement avec la taille du poisson. D'après BLAXTER (1969), la limite supérieure de la vitesse de croisière de la majorité des espèces se situerait entre 2 et $3 \mathrm{~L} / \mathrm{s}$, L étant la longueur du poisson exprimée en mètre. Pour les salmonidés, elle atteindrait de 3 à $4 \mathrm{~L} / \mathrm{s}$. On peut donc admettre, pour le saumon, des vitesses de croisière de l'ordre de $2 \mathrm{~m} / \mathrm{s}$ à $3 \mathrm{~m} / \mathrm{s}$; pour la truite fario, elles se situeraient entre $0.50 \mathrm{~m} / \mathrm{s}$ et $1 \mathrm{~m} / \mathrm{s}$ suivant la taille de l'individu alors qu'elles seraient de l'ordre de $0.45 \mathrm{~m} / \mathrm{s}$ à $0.60 \mathrm{~m} / \mathrm{s}$ pour la majorité des juvéniles de saumon en période de dévalaison. Le modèle empirique dont les résultats sont donnés sur les figures 1 à 3 a tendance à sousestimer les valeurs des vitesses de croisière des poissons de petite taille. 


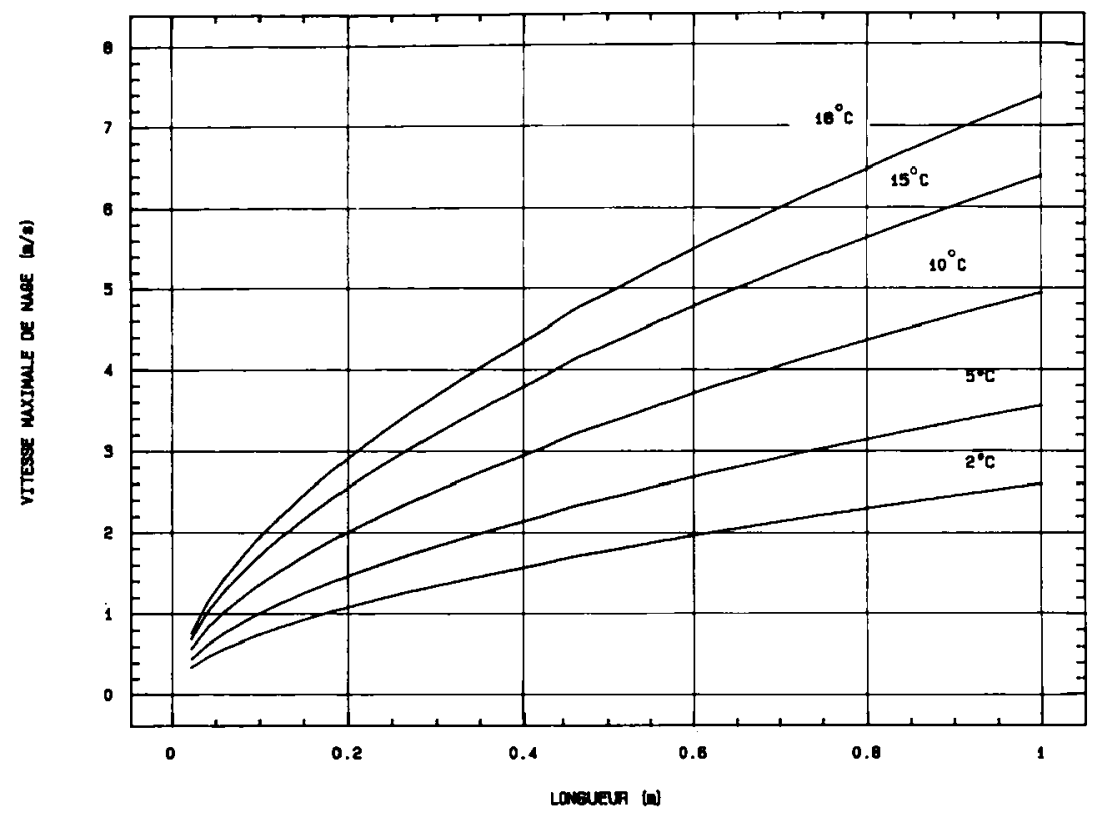

Figure 1 : Vitesse maximale de nage en fonction de la taille du poisson et de la température pour les salmonidés (d'après BEACH, 1984).

Figure 1 : Maximum swimming speed vs. fish length and temperature for salmonids (from BEACH, 1984).

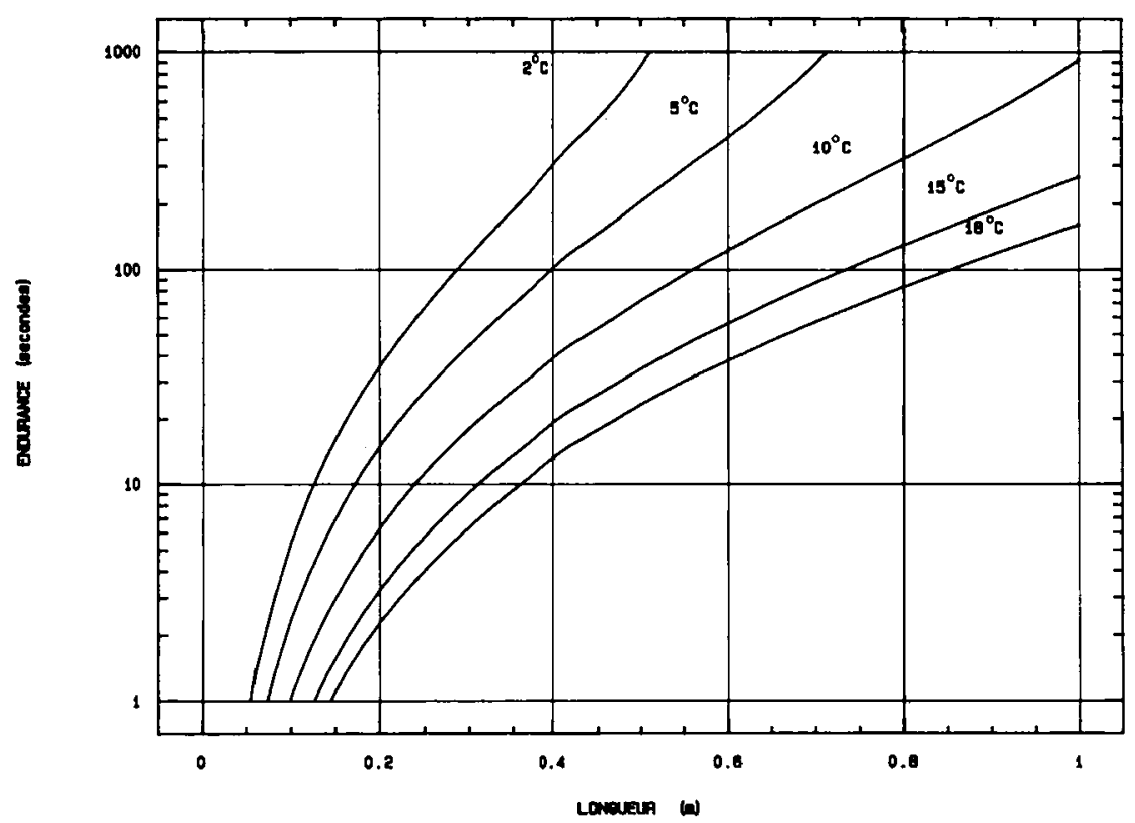

Figure 2 : Endurance à la vitesse maximale de nage en fonction de la taille du poisson et de la température pour les salmonidés (d'après $\mathrm{BEACH}, 1984$ ).

Figure 2 : Endurance at maximum swimming speed vs. fish length and temperature for salmonids (from BEACH, 1984). 


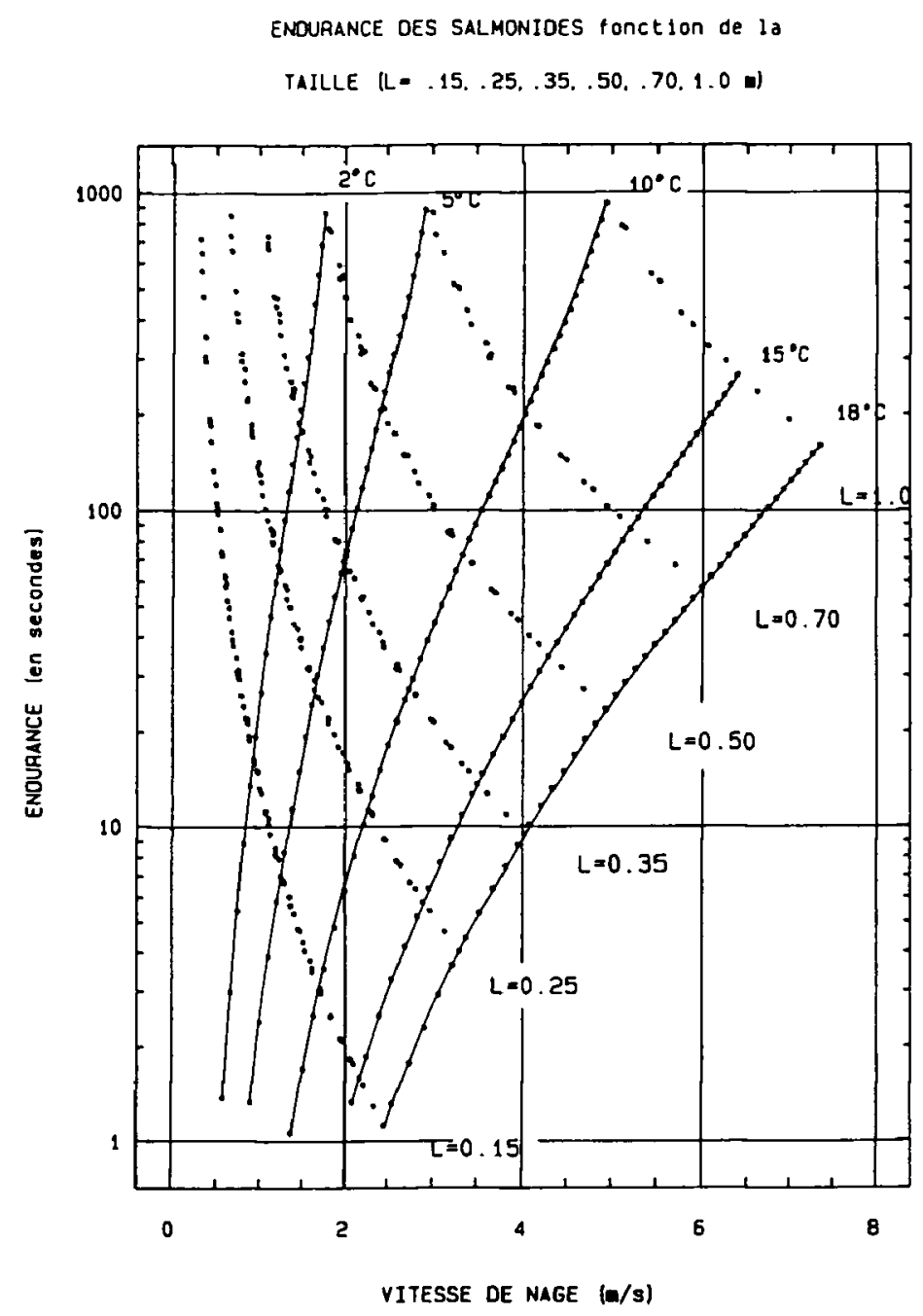

Figure 3 : Vitesse de nage et endurance en fonction de la température et de la taille des poissons pour les salmonidés.

Figure 3 : Swimming speed and endurance vs. fish length and temperature for salmonids.

\subsection{Distances maximales franchissables dans un écoulement de vitesse donnée}

A partir des relations empiriques précédentes, on peut déterminer la distance maximale $D$ pouvant être parcourue par un poisson dans un écoulement de vitesse donnée U.

Cette distance est donnée par l'expression :

$$
\mathrm{D}=(\mathrm{V}-\mathrm{U}) \mathrm{T}
$$

où $T$ est l'endurance (exprimée en secondes) de l'individu nageant à la vitesse $\mathrm{V}$.

On a porté à titre d'exemple, sur la figure 4 , les distances maximales pouvant être parcourues par des salmonidés de longueurs $0.35 \mathrm{~m}$ et $0.20 \mathrm{~m}$.

Ces courbes sont relativement proches des courbes semi-empiriques portées à la figure 5 proposées par ZIEMER (1961) et EVANS et JOHNSTON (1980), sauf que ces dernières ne prennent pas en compte le facteur thermique. 


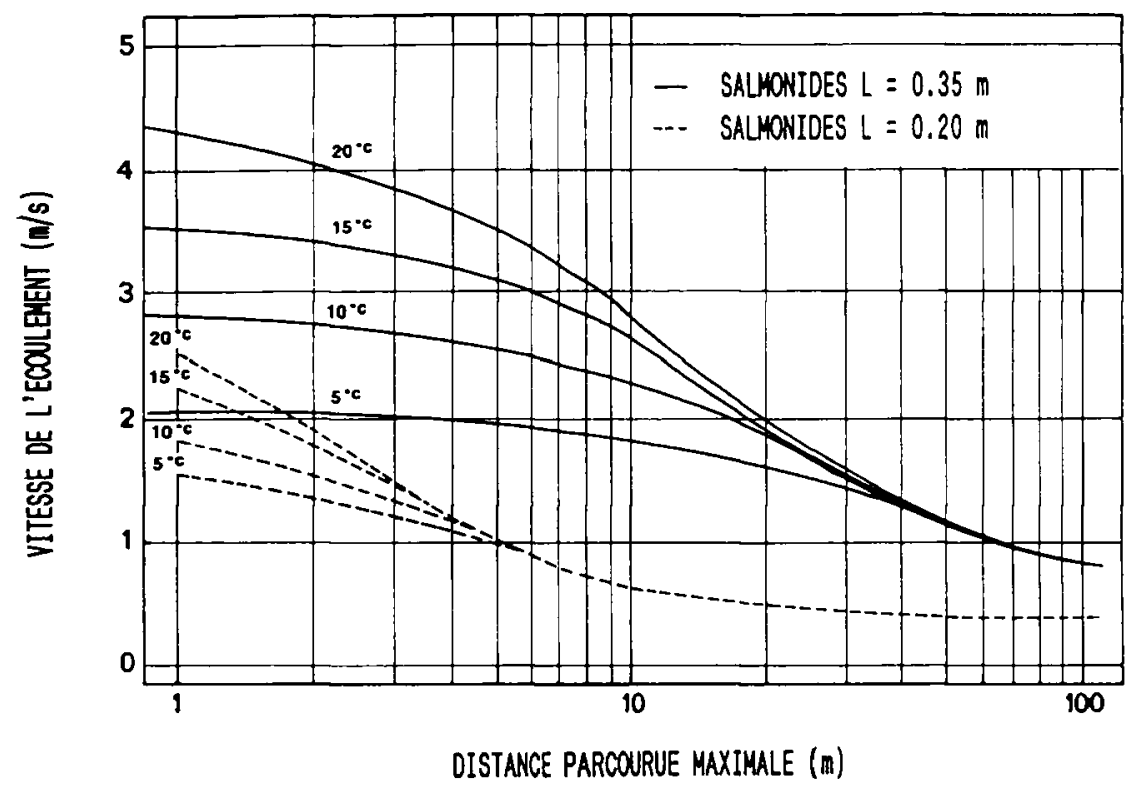

Figure 4 : Distance maximale parcourue en fonction de la vitesse de l'écoulement et de la température pour deux tailles de salmonidés.

Figure 4 : Maximum swimming distance vs. water velocity and temperature for two lengths of salmonids.

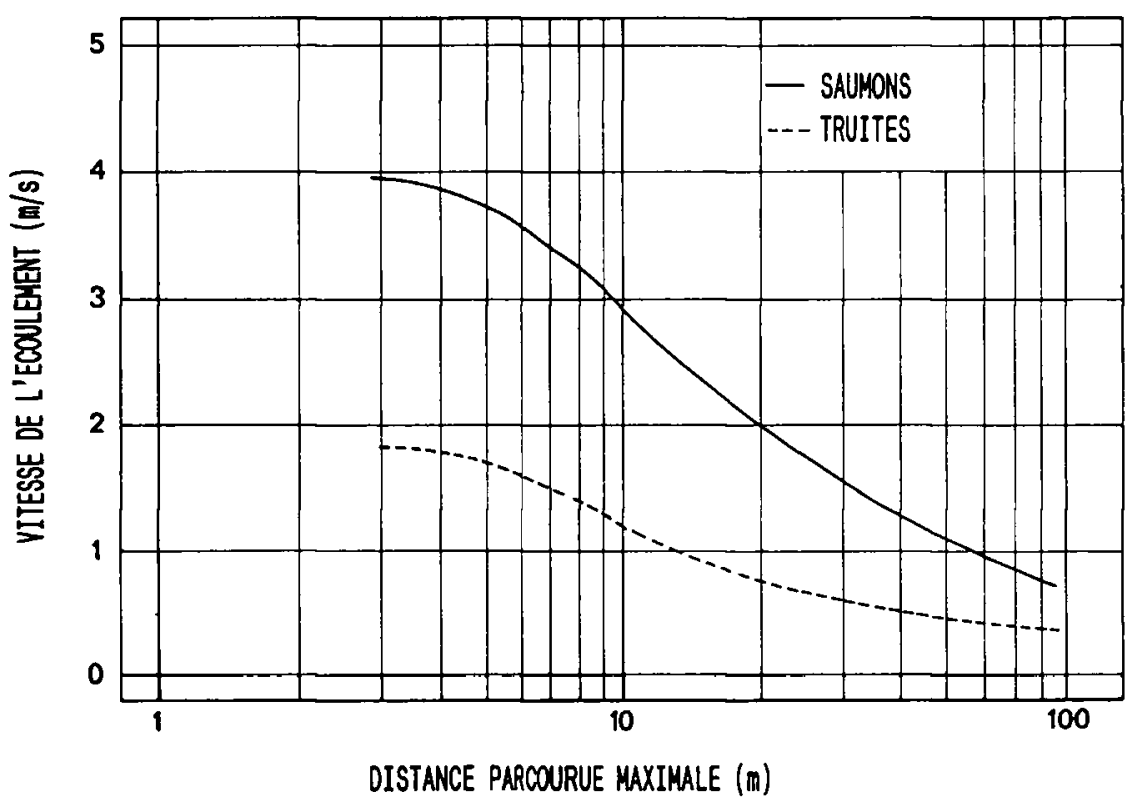

Figure 5 : Capacité de nage du saumon et de la truite (d'après ZIEMER, 1961 et EVANS et JONHSTON, 1980).

Figure 5 : Swimming capacity of migrating salmon and trout (from ZIEMER, 1961 and EVANS and JONHSTON, 1980). 


\subsection{Capacités de saut}

Certaines espèces, en particulier les salmonidés, sont capables de franchir un obstacle en sautant, à condition que le poisson trouve au pied de l'obstacle des conditions lui permettant de prendre son appel.

Le mouvement du poisson effectuant un saut peut être assimilé à la trajectoire d'un projectile. L'équation de la trajectoire peut s'exprimer sous la forme :

$$
\begin{gathered}
X=(\text { Vo cos } a) t \\
Y=(\text { Vo sina }) t-0.5 g t^{2}
\end{gathered}
$$

où $X$ et $Y$ sont les distances horizontales et verticales parcourues par le projectile en l'occurrence le poisson - , Vo la vitesse initiale, a l'angle d'incidence avec le plan horizontal et $\mathrm{g}$ l'accélération de la pesanteur. La trajectoire du poisson est parabolique, la hauteur maximale atteinte par le poisson dépend de sa vitesse initiale et de l'incidence du saut au départ:

$$
\mathrm{Ymax}=\left(\mathrm{Vo}_{\mathrm{m}} \sin \mathrm{a}\right)^{2} / 2 \mathrm{~g}
$$

La distance horizontale correspondant à cette hauteur maximale Xmax est donnée par l'expression :

$$
X \max =V_{o^{2}} \operatorname{cosa} \sin \mathrm{a} / \mathrm{g}
$$

On a porté sur un même graphique (Fig. 6) les trajectoires de saut théoriques d'un saumon en fonction de l'angle d'incidence à sa sortie de l'eau et de la température. Ce graphique met en évidence le rôle prépondérant de la température sur la hauteur franchissable par le saut.

On peut cependant noter (POWERS et OSBORN, 1985) qu'en toute rigueur il conviendrait d'ajouter à cette hauteur Ymax une hauteur correspondant à une forte fraction de la longueur du poisson dans la mesure où celui-ci utilise sa force propulsive jusqu'au moment où sa nageoire caudale quitte la surface de l'eau. D'autre part, l'équation précédente ne tient pas compte de la composante ascendante de la vitesse existant au pied d'une chute, dont le poisson peut profiter. Les valeurs de saut obtenues par la formule ci-dessus sont donc des valeurs conservatoires.

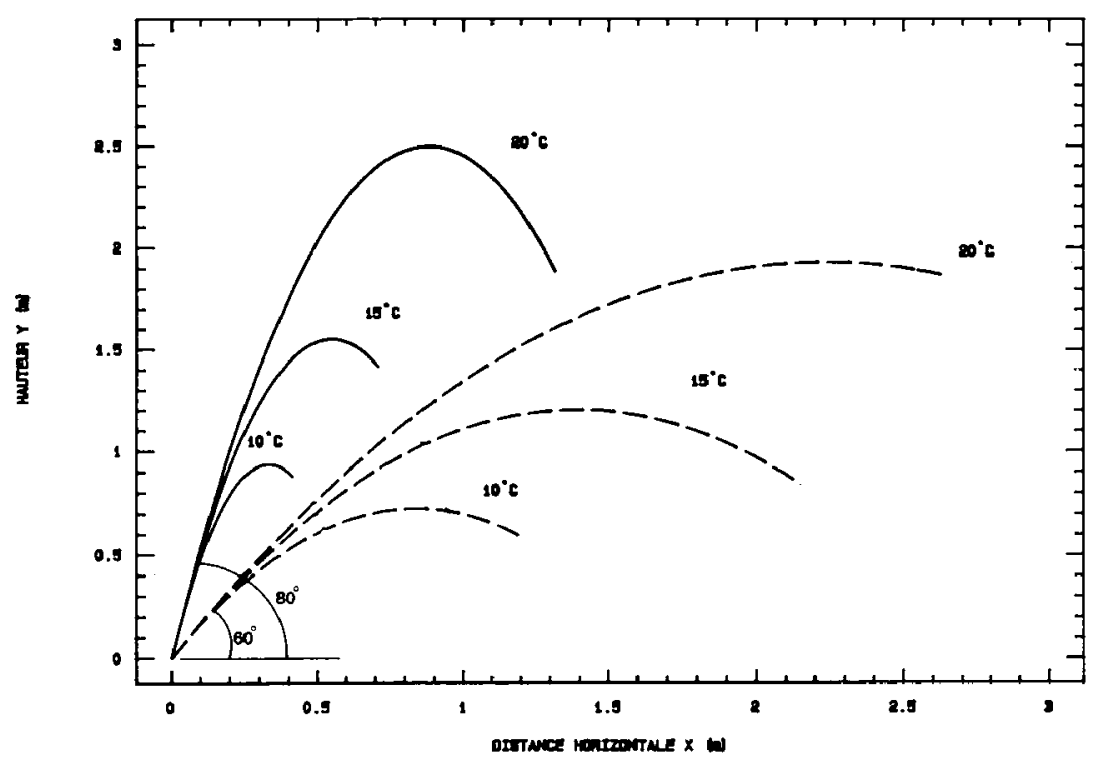

Figure 6 : Trajectoires de saut théoriques pour un saumon de $0.80 \mathrm{~m}$ de longueur en fonction de la température et de l'angle d'incidence du saut.

Figure 6 : Theorical leaping curves vs. temperature and angle of leap from water surface for salmon (length $0.80 \mathrm{~m}$ ). 


\subsection{Capacités de nage et dispositifs de franchissement}

Les vitesses maximales proposées plus haut sont relatives à des poissons en excellente condition physique (pas de blessures, temps de séjour en eau douce réduit pour les grands migrateurs, etc...). Par ailleurs, l'endurance doit être prise comme une durée d'effort maximale provoquant l'épuisement total du poisson. En pratique, il convient de se situer bien en deçà de ces limites pour ce qui est de l'effort à demander aux migrateurs dans un dispositif de franchissement.

L'utilisation de la vitesse moyenne d'un écoulement (vitesse moyenne débitante par exemple, qui est le rapport du débit à la section de l'écoulement), comme seul critère de "franchissabilité" pour un obstacle ou de dimensionnement pour un dispositif de franchissement est cependant délicate :

- d'une part les poissons sont capables de percevoir de très faibles variations des vitesses de l'écoulement et cherchent généralement les zones les plus favorables à leur progression (voisinage des parois, zones de décollement, etc...),

- d'autre part, lorsque dans un écoulement les fluctuations spatio-temporelles de vitesses deviennent importantes (écoulements tourbillonnaires par exemple), l'énergie nécessaire au poisson pour franchir une certaine distance peut devenir beaucoup plus importante que celle requise pour parcourir la même distance dans un écoulement plus régulier à filets parallèles ayant même vitesse moyenne, et cela d'autant plus que les vitesses maximales de l'écoulement se rapprochent des vitesses de pointe du poisson.

C'est le cas en particulier de l'écoulement dans les passes à ralentisseurs caractérisé par des champs de vitesses fortement tourbillonnaires et tridimensionnels et dans lequel le poisson est susceptible d'emprunter certaines zones particulières à plus faible vitesse lors de sa progression.

Les abaques donnés plus haut mettent en évidence que les espèces de petite taille ont des vitesses de pointe limitées et qu'elles ne peuvent soutenir ces vitesses ou des vitesses légèrement inférieures qu'un très bref instant (quelques secondes).

Seules les passes à bassins successifs pourront convenir à ces espèces : dans ce type de passe le poisson n'a à fournir d'effort qu'au passage de l'échancrure, de la fente ou de l'orifice sur une distance n'excédant pas quelques décimètres. Le poisson peut d'autre part éviter les zones à vitesses maximales en profitant au maximum du décollement des lames d'eau.

Les passes à ralentisseurs de fond, dans lesquelles les vitesses ponctuelles descendent dans le meilleur des cas (passe faiblement alimentée, ralentisseurs hydrauliquement "efficaces") à des valeurs voisines de $1 \mathrm{~m} / \mathrm{s}$ et qui sont très sensibles aux variations du niveau d'eau amont, ne doivent être utilisées que pour les migrateurs de grande taille.

Les passes à ralentisseurs plans ou de type FATOU dans lesquelles les vitesses peuvent descendre à des valeurs voisines de $0.7 \mathrm{~m} / \mathrm{s}-0.8 \mathrm{~m} / \mathrm{s}$ sont susceptibles d'assurer le passage d'espèces ou d'individus de plus petite taille à condition que la longueur des volées reste inférieure à 6-8 mètres et que la dimension des ralentisseurs soit réduite et ramenée à l'échelle du poisson.

\section{ECLAIRAGE DES DISPOSITIFS DE FRANCHISSEMENT}

Lorsqu'on est confronté au problème de la couverture d'une passe - ou à celui du franchissement d'une buse par les migrateurs - se pose la question de savoir si un éclairage, qu'il soit naturel ou artificiel, est nécessaire.

Un certain nombre d'observations effectuées sur les salmonidés (AITKEN et al., 1966 ; ROGERS et CANE, 1979) indiquent que le poisson peut franchir une passe ou un canal en tunnel d'une très grande longueur sans qu'il soit nécessaire de l'éclairer.

D'après BELL (1986), la progression des migrateurs n'est pas ralentie par l'obscurité. 
Des expérimentations effectuées sur la côte Ouest des USA (LONG, 1959) ont montré que si le migrateur (truite steelhead) hésitait plus longtemps à s'engager dans une passe obscure, il remontait celle-ci plus rapidement que dans la même passe éclairée (deux minutes par bassin contre plus de huit).

Des expérimentations ont été effectuées en vue d'inciter les migrateurs à emprunter des passes à poissons la nuit en éclairant celles-ci. Tous les essais se sont montrés peu concluants : au barrage de The Dalles (côte Ouest des USA), moins de $10 \%$ des migrateurs empruntent la passe entre $20 \mathrm{~h}$ et $4 \mathrm{~h}$, que la passe soit éclairée ou non. Au barrage de McNary, les essais ont montré que le poisson n'entrait pas dans la passe de nuit, quelles que soient les conditions d'éclairage ; par contre, cet éclairage permettait aux poissons ayant pénétré dans la passe avant la tombée du jour de franchir la passe dans son intégralité (FIELDS, 1966).

Sur certains aménagements (Grand Sault, rivière Madeleine, Québec), l'observation a montré que le saumon atlantique éprouvait de la répulsion à pénétrer dans la partie souterraine d'une passe à bassins successifs, la transition lumière-obscurité étant brutale : la passe ne fonctionne correctement que lorsqu'elle est éclairée.

Sur l'aménagement de Tuilières sur la Dordogne, la section de sortie de l'ascenseur à poissons est constituée de neuf bassins souterrains : les poissons piégés dans la cuve de l'ascenseur sont déversés dans le bassin aval d'une passe couverte. L'éclairage artificiel de cette passe s'est révélé indispensable pour permettre son franchissement par les aloses qui avaient tendance à rester bloquées dans les bassins aval de l'ouvrage, les mieux éclairés naturellement (DARTIGUELONGUE et al., 1992).

A l'inverse, l'éclairage d'un dispositif de franchissement peut inhiber la migration nocturne de certaines espèces lucifuges qui peuvent se trouver partiellement voire totalement bloquées.

Des expérimentations ont été effectuées pour tenter de mettre en évidence l'influence de l'éclairage sur le passage de diverses espèces de saumons et truites du Pacifique dans des conduites de diamètres $0.60 \mathrm{~m}$ et $0.90 \mathrm{~m}$ (SLATICK, 1970). Les résultats ont montré que toutes les espèces pouvaient franchir ces conduites sans éclairage. Sur les quatre espèces testées (chinook, coho, sockeye et truite steelhead), seule la truite steelhead semblait bénéficier de façon significative (en terme de $\%$ de passage) de l'éclairage des conduites. La vitesse de passage semblait cependant plus importante lorsque le tuyau était éclairé pour la truite steelhead, les saumons sockeye et coho, alors que c'était l'inverse pour le saumon chinook.

En conclusion, il paraît donc préférable, lorsqu'on est obligé d'enterrer ou de couvrir une passe, d'éviter une brusque transition entre l'intensité lumineuse du milieu extérieur et celle de la passe ou de la buse en éclairant d'une façon ou d'une autre (éclairage artificiel, fenêtres, évasement de l'entrée) le premier tronçon de l'ouvrage. Certains auteurs (METSKER, 1968) recommandent de végétaliser l'entrée et la sortie de l'ouvrage (dans le cas de buses) pour réduire les gradients d'éclairement à l'entrée et à la sortie.

Si l'éclairage d'un dispositif dans sa totalité n'apparaît pas, à l'expérience, comme un facteur prépondérant conditionnant son franchissement par la majorité des espèces (sauf peut-être pour certaines espèces comme l'alose), il peut cependant être retenu dans tous les cas comme un facteur de sécurité lors de la conception d'un ouvrage.

\section{NOTION D'OBSTACLE A LA MIGRATION}

On a souvent tendance à associer la notion d'obstacle à la migration à la hauteur de la chute. La réalité est beaucoup plus complexe : le fait qu'un obstacle soit franchissable ou non dépend des conditions hydrodynamiques sur et au pied de l'obstacle (vitesses, tirants d'eau, configuration des jets, aération, turbulence...) en relation avec les capacités de nage et de saut des espèces considérées.

Les conditions hydrodynamiques dépendent à la fois de la géométrie de l'ouvrage (hauteur et profil du barrage, pentes, longueurs) et des débits qui y transitent, c'est-à-dire des conditions hydrologiques en période de migration ainsi qu'éventuellement de la gestion de l'ouvrage. 
On a porté sur la figure 7 plusieurs configurations d'obstacles ayant même hauteur de chute. Si les capacités de nage du poisson le permettent, l'obstacle (a) est franchissable. En (b), la lame d'eau insuffisante sur le parement aval ne permet pas la nage du poisson. En (c), la présence d'un radier en pied de barrage et l'absence de fosse d'appel rendent l'obstacle infranchissable quelles que soient les capacités de nage du poisson. En (d), la présence d'une rehausse bloque le poisson au niveau de la rupture de pente.

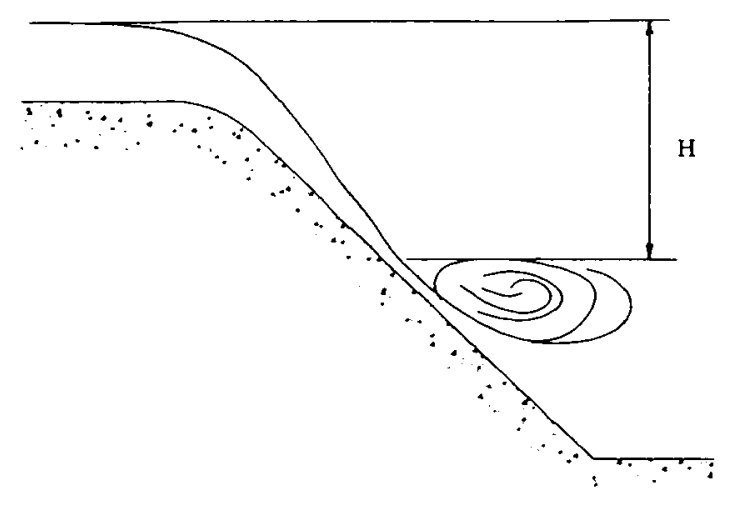

a-

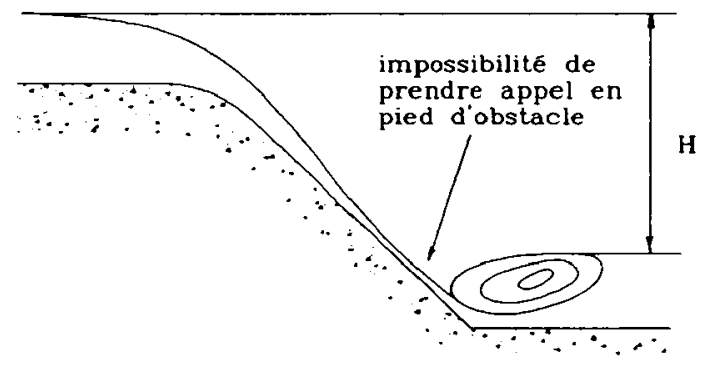

c-

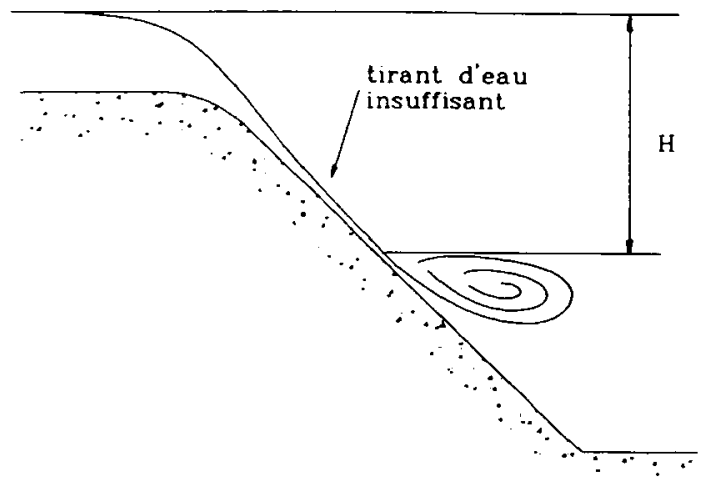

b-

\section{Figure 7 : Schéma illustrant l'influence de la configuration d'un obstacle sur sa franchissabilité.}

\section{Figure 7 : Schematic plan illustrating the influence of the profile of a weir on difficulty in passage.}

On a vu que les capacités de nage et de saut dépendaient de l'espèce, de la taille des individus, de leur état physiologique, ainsi que des conditions thermiques ambiantes. La franchissabilité d'un ouvrage est donc liée à sa géométrie, aux espèces considérées et aux conditions hydrologiques et thermiques en période de migration. Pour ces diverses raisons, la plupart des auteurs considèrent que des blocages peuvent intervenir pour des chutes ne dépassant pas $0.50 \mathrm{~m}$ à $0.60 \mathrm{~m}$ pour les grands salmonidés migrateurs, voire moins pour d'autres espèces à capacité de nage plus réduites comme l'anguille.

La franchissabilité d'un obstacle doit être considérée pour chaque espèce migratrice présente dans le cours d'eau. Pour une espèce donnée, un obstacle sur un cours d'eau 
peut être total, c'est-à-dire infranchissable en permanence pour tous les individus. II peut être partiel, c'est-à-dire infranchissable pour certains individus. II peut être temporaire, c'est-à-dire totalement infranchissable à certaines périodes de l'année (sous certaines conditions hydrologiques ou thermiques). Les cas très fréquents sont ceux d'obstacles de faible hauteur infranchissables en étiage à cause des trop faibles tirants d'eau sur le parement aval ne permettant pas la nage des poissons. Certains ouvrages peuvent être infranchissables par faible température, le poisson n'ayant pas alors les capacités de nage suffisantes pour le franchir.

II ne faut pas sous-estimer l'impact négatif des obstacles temporaires qui retardent les poissons dans leur migration et peuvent l'obliger à stabuler dans des zones peu propices dans la partie basse des cours d'eau, ou provoquer des blessures à la suite de tentatives de franchissement répétées et infructueuses.

\section{BIBLIOGRAPHIE}

AITKEN P.L., DICKERSON L.H., MENZIES W.J.M., 1966. Fish passes and screens at water power works. Proc. Inst. Civ. Eng., $35: 29-57$.

BEACH M.H., 1984. Fish pass design. Criteria for the design and approval of fish passes and other structures to facilitate the passage of migratory fishes in rivers. Ministry of Agriculture, Fisheries and Food, Lowestoft, Fish. Res. Tech. Rep. 78, 45 p.

BELL M.C., 1986. Fisheries handbook of engineering requirements and biological criteria. Fish. Eng. Res. Prog., U.S. Army Corps of Eng., North Pacific Div., Portland, Oregon, 290 p.

BLAXTER J.H.S., 1969. Swimming speeds of fish. FAO Fish. Rep., 62(1) : 69-100.

DARTIGUELONGUE J., LARINIER M., TRAVADE F., 1992. Etude du comportement de l'alose dans la passe à poissons à l'usine de Tuilières sur la Dordogne. Rapp. CSPEDF, $55 \mathrm{p}$.

EVANS W.A., JOHNSTON F.B., 1980. Fish migration and fish passage : a practical guide to solving fish passage problems. USDA Forest Serv., Region 5, $43 \mathrm{p}$.

FIELDS P.E., 1966. Final report on migrant salmon light guiding studies at Columbia rivers dams. Fish. Eng. Res. Prog., U.S. Army Corps of Eng., North Pacific Div., Portland, Oregon, $266 \mathrm{p}$.

LONG C.W., 1959. Passage of salmonids through a darkened fishway. U.S. Fish and Wildlife Serv., Spec. Sci. Rep. Fisheries 300,9 p.

METSKER H.E., 1970. Fish versus culverts, some considerations for resource managers. USDA Forest Serv., Ogden, Utah, Eng. Tech. Rep. ETR-7700-5, 22 p.

POWERS P., OSBORN J., 1985. Analysis of barriers to upstream fish migration. U.S. Dept. of Energy, Bonneville Power Adm., Div. of Fish and Wildlife, Final Project Rep., 120 p.

ROGERS A., CANE A., 1979. Upstream passage of adult salmon through an unlit tunnel. Fish. Mgmt., $10(2): 87-92$.

SLATICK E., 1970. Passage of adult salmon and trout through pipes. U.S. Fish and Wildlife Serv., Spec. Sci. Rep. Fisheries 92, 18 p.

WARDLE C.S., 1975. Limit of fish swimming speed. Nature, London, 225 : 725-727.

WARDLE C.S., 1980. Effects of temperature on the maximum swimming speed of fishes. pp. 519-531. In "Environmental Physiology of Fishes", Plenum Press (Ed.), New York and London, $723 \mathrm{p}$.

WEBB P.W., 1975. Hydrodynamics and energetics of fish propulsion. Bull. Fish. Res. Bd. Can., 190, $158 \mathrm{p}$.

ZHOU Y., 1982. The swimming speed of fish in towed gears, a reexamination of the principles. Dept. of Agriculture and Fisheries for Scotland, Work. Pap. 4, 55 p.

ZIEMER G.L., 1961. Fish transport in waterways. Alaska Dept. of Fish and Game, 10 p. 